\title{
Technical Issues of Blood Pressure Measurement and Its Impact
}

\author{
Author \\ Ashraf Safiya Manzil \\ MD DM FESC FSCAI FACC \\ Professor of Cardiology, Pariyaram Medical College, Kerala, India \\ Email:ashsmfz@yahoo.com
}

Diagnosis of hypertension is based on bolod pressure (BP) measurements with a sphygmomanometer. BP levels of 140/ $90 \mathrm{~mm} \mathrm{Hg}$ and higher are arbitrarily considered hypertensive, where as BP levels of $139 / 89 \mathrm{mmHg}$ or less are considered normal or pre hypertensive ${ }^{(1)}$. Some technical problems in BP measurements may give erroneously elevated BP levels and wrong diagnosis of hypertension. so it is great challenge to determine BP accurately.

Although the use of mercury sphygmomanometer is regarded as the 'gold standard' for office blood pressure measurement, widespread ban in use of mercury devices has diminished their role in hospital settings. Alternative methods such as automated electronic devices have gained increased popularity. The preferred location of measurement is the upper arm, but errors may occur because of changes in the position of the arm (2). Other technical sources of error include inappropriate cuff size and too rapid deflation of the cuff. It is common among obese people in whom the inflatable bladder may be too small for the arm size, thereby leading to "Cuff hypertension".

Clinic readings may be unrepresentative of the patient's true blood pressure because of the white coat effect, which is defined as the difference between the clinic readings and the average daytime blood pressure. Patients with elevated clinic pressure and normal daytime pressure are said to have white coat hypertension, which is often explained by state anxiety or conditioned response. There are three commonly used methods for measuring blood pressure for clinical purposes: clinic readings, self-monitoring by the patient at home, and 24-hour ambulatory readings (3). Self-monitoring is generally carried out using electronic devices that work on the oscillometric technique. Although standard validation protocols exist, many devices on the market have not been tested for accuracy. Such devices can record blood pressure from the upper arm, wrist, or finger, but the arm is preferred.

Twenty-four-hour ambulatory monitoring is the best predictor of cardiovascular risk in the individual patient and is the only technique that can describe the diurnal rhythm of blood pressure accurately. Ambulatory monitoring is mainly used for diagnosing hypertension, whereas selfmonitoring is used for following the response to treatment. Different techniques of blood pressure measurement may be preferred in certain situations. In infants the ultrasound technique is 
best, whereas in pregnancy and after exercise the diastolic pressure may be hard to measure using the conventional auscultatory method ${ }^{(4)}$. To avoid overestimation of diastolic pressure, it is necessary to confirm the disappearance of the sounds by listening as the pressure falls another 10 to $20 \mathrm{~mm}$ $\mathrm{Hg}$. In obese subjects it is important to use a cuff of the correct size.

In persons older than 50 years, systolic blood pressure (BP) of more than $140 \mathrm{~mm} \mathrm{Hg}$ is a much more important cardiovascular disease (CVD) risk factor than diastolic $\mathrm{BP}{ }^{(5)}$. The risk of CVD, beginning at $115 / 75 \mathrm{~mm} \mathrm{Hg}$, doubles with each increment of 20/10 mm Hg; individuals who are normotensive at 55 years of age have a $90 \%$ lifetime risk for developing hypertension. Individuals with a systolic BP of 120 to $139 \mathrm{~mm}$ $\mathrm{Hg}$ or a diastolic BP of 80 to $89 \mathrm{~mm} \mathrm{Hg}$ should be considered as prehypertensive and require health-promoting lifestyle modifications to prevent CVD. Most patients with hypertension will require 2 or more antihypertensive medications to achieve goal BP $(<140 / 90 \mathrm{~mm} \mathrm{Hg}$, or $<130 / 80 \mathrm{~mm} \mathrm{Hg}$ for patients with diabetes or chronic kidney disease). If $\mathrm{BP}$ is more than $20 / 10 \mathrm{~mm} \mathrm{Hg}$ above goal BP, consideration should be given to initiating therapy with 2 agents, 1 of which usually should be a thiazide-type diuretic. The most effective therapy prescribed by the most careful clinician will control hypertension only if patients are motivated. Motivation improves when patients have positive experiences with and trust in the clinician. Empathy builds trust and is a potent motivator. Hypertension is often asymptomatic and recording blood pressure is opportunistic. Many of these patients are unaware of their condition and therefore remain untreated. Untreated or poorly controlled hypertension and left ventricular hypertrophy (LVH) are risk factors for cardiovascular diseases (CVD), a major cause of morbidity and mortality, and sudden death.The lack of awareness of the disease results in worse outcomes.

A number of hypertensives may present for the first time with target-organ damage (TOD) involving various organs ${ }^{(6)}$. Therefore at initial diagnosis, they already have hypertensive heart disease (HHD); some with LVH, while some have frank congestive heart failure (CHF). CHF is a lethal disease with a poor prognosis and reduced life expectancy. It imposes a large health, economic and social burden on the patient, his/her family and the community at large, and it usually affects males and females in their productive middle years. So, It is paramount importance for actual measurement of blood pressure in a person who especially has risk factors.

\section{References}

1. Chobanian AV, Bakris GL, Black HR, et al; and the National High Blood pressure Education Programme Coordinating Committee: The Seventh Report of the Joint National Committee on Prevention, Detection, Evaluation, and Treatment of High Blood PressureJNC 7 report. JAMA. 2003; 289: 2560- 2572

2. O' Brien E, Asmar R, Beilin L, et al. Practice guidelines of the European Society of Hypertension for clinic, ambulatory and self blood pressure measurement. J Hypertens. 2005: 23:697-701.

3. Cooper R, Puras A, Tracy J, et al. Evaluation of an electronic blood pressure device for epidemiological studies. Blood Press Monit. 1997;2:35.

4. Padfield PL, Jyothinagaram SG, Watson $\mathrm{DM}$, et al. Problems in the measurement of blood pressure. J Hum Hypertens. 1990;4 (Suppl 2):3.

5. 89. Verdecchia P, Angeli F, Gattobigio R, et al. Impact of blood pressure variability on cardiac and cerebrovascular complications in hypertension. Am J Hypertens. 2007;20:154.

6. Familoni OB, Alebiosu CO, Odusan A, Raimi A. Factors influencing target organ damage among hypertensive patients. Trop Cardiol. 2003;29:21-24. 